\title{
Scleral thinning after eye-whitening surgery
}

\author{
Takeshi Ide, 1,2 Yoshiyuki Ichihashi, ${ }^{1,2}$ Ikuko Toda, ${ }^{1,2}$ \\ Teruki Fukumoto, ${ }^{1,3}$ Kazuo Tsubota ${ }^{2}$ \\ ${ }^{1}$ Minamiaoyama Eye Clinic, Tokyo, Japan; '2Department of Ophthalmology, School \\ of Medicine, Keio University, Tokyo, Japan; ${ }^{3}$ Department of Ophthalmology, \\ National Defense Medical College, Saitama, Japan
}

\begin{abstract}
We report the case of a 32-year-old male patient who presented with conjunctival redness after multiple bilateral eye-whitening surgeries. Slit-lamp examination revealed scleral melting, avascular areas, and demarcation lines in both eyes. Considering the treatment history and the clinical findings of the patient, we conclude that the removal of the conjunctiva and Tenon's layer and use of mitomycin $C$ is not desirable for a healthy ocular surface.
\end{abstract}

Key words: eye-whitening surgery, MMC, mitomycin C, scleral melt, sclera, conjunctiva

\section{Introduction}

The conjunctiva is a translucent mucous membrane that covers the inner surface of the eyelids and sclera and extends to the limbus on the surface of the globe. It plays a critical role in maintaining the integrity of the ocular surface; also, it serves as the source of the tear mucous layer ${ }^{1}$ and assists the immunity system. ${ }^{2}$

With aging, the conjunctiva loses transparency due to keratinization; changes to these layers include yellowing, browning, pinguecula, or vascularization due to years of exposure to ultraviolet light, wind, and dust. These changes are not uniform; they tend to be more pronounced in the interpalpebral fissure, as it is frequently exposed to the outside environment.

Although we, ophthalmologists, feel that these changes due to aging are normal, patients may be uncomfortable with them. Hence, they undergo eye-whitening surgeries in order to whiten and brighten the eye. An eye-whitening surgery involves the removal of the hyperemic conjunctiva and Tenon's layer and the intra-operative application of mitomycin $C(M M C)$ to the bare sclera. This helps in removing excessive red veins, yellow growths, and areas of brown pigmentation. In this case report, we present a case of bilateral scleral thinning after multiple eye-whitening surgeries.

\section{Case report}

A 32-year-old Japanese man had chronic severe red eyes, which caused mental distress. He underwent the first eye-whitening surgery with intraoperative MMC in 2009. Postoperatively, he was on a steroid taper, antibiotic, and limited $0.02 \%$

Correspondence: Takeshi Ide, MD, PhD, Minamiaoyama Eye Clinic, Renai Aoyama Building 4F, 3-3-11, Kitaaoyama, Minato-ku, Tokyo 107-0061, Japan.

E-mail: teyede@minamiaoyama.or.jp 
MMC drops (four times a day for seven days). The conjunctiva regrew and covered the sclera and Tenon's layer. Cosmesis was improved, but excessive vessels regrew around the limbus, which was not acceptable to the patient. Hence, he desired a second procedure that was performed in early 2010. MMC was not applied intraoperatively. The medications after the second surgery were the same as those after the first one, including $0.02 \%$ MMC drops (four times a day for seven days).

Regardless of the doctor's warning against the increased risk of scleral melt, the patient desired further improvement in the perilimbal vessels. Hence, a third surgery was performed later in 2010, and no intraoperative MMC was applied. The same postoperative regimen was introduced, and it included MMC, as detailed above.

The patient first visited us in November 2011 and claimed that he had some dark areas on the sclera. Slit-lamp examination revealed focal areas of nasal and temporal scleral melting in the right eye and nasal thinning in the left eye, both with overlying plaques. In addition, avascular areas and demarcation lines were observed in the midperipheral regions (Fig. 1). However, prolapsed uveal tissue was not observed. The anterior chamber and posterior segment were quiet. The operative record from the surgeon did not indicate whether the area of scleral melting corresponded to areas of conjunctival regrowth after the second surgery.

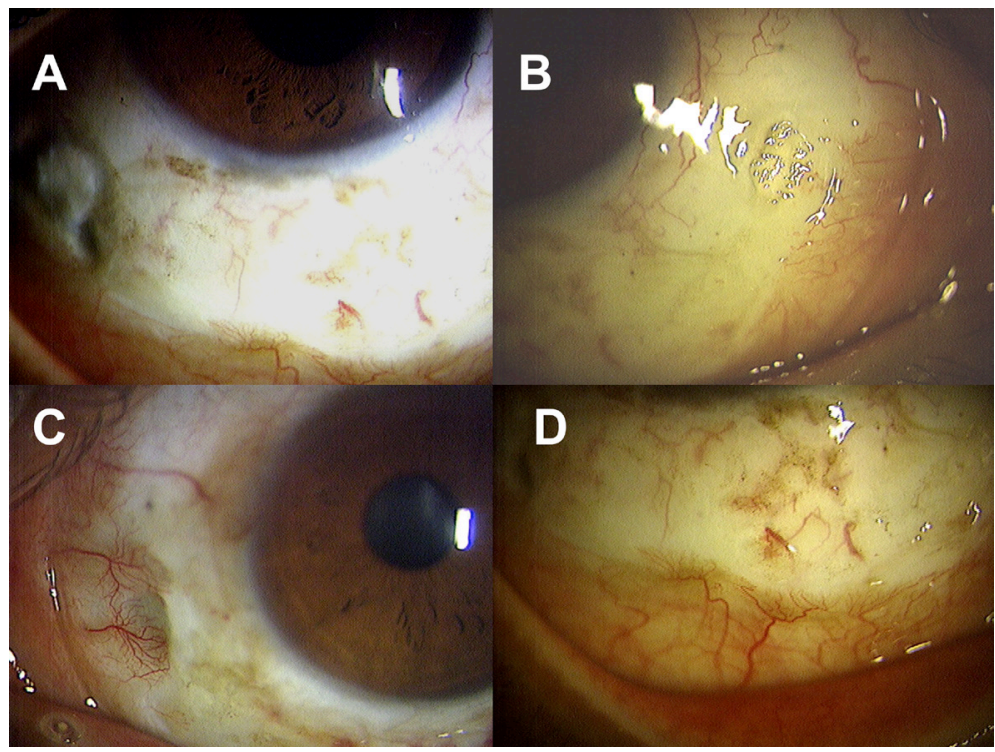

Fig. 1. Ocular manifestations after eye-whitening surgery. The areas with scleral thinning can be observed in the temporal area (A), nasal area in the right eye (B), and nasal area in the left eye (C). In addition, obvious demarcation lines are seen in the mid-peripheral area (A-D).

The patient claimed that he did not have general diseases. After the first visit, he did not come back to us for follow-ups in spite of our advice, and we could not take any investigations like blood tests to exclude autoimmune disorders. His 
uncorrected visual acuity and best spectacle corrected visual acuity were respectively, 0.9 and 1.2 in the right eye, and 1.2 and 1.5 in the left eye. The intraocular pressure (IOP) was $11 \mathrm{mmHg}$ in the right eye and $13 \mathrm{mmHg}$ in the left eye.

\section{Discussion}

In the current case report, we present a complication of scleral melting after repeated eye-whitening surgeries. Other than this complication, the patient's eye function was normal.

Corneo-scleral melt is a severe condition characterized by progressive tissue thinning that often leads to perforation. It may be initiated by various factors such as systemic or local autoimmune diseases, chemical or thermal burns, dry eyes, infections, or previous ocular surgeries. Further, this melting can be caused by a delayed-onset hypersensitivity response to localized ischemia that involves the episcleral blood vessels. ${ }^{3-5}$ With respect to surgeries, it is reported mostly after pterygium surgery with bare sclera technique, particularly in cases where adjunctive radiation or chemotherapy is used..$^{6-15}$

MMC acts as an alkylating agent and a potent fibroblast inhibitor, which can cause irreversible damage to the cell. ${ }^{16-17}$ In addition, it inhibits the migration of fibroblasts and the synthesis of collagen, and therefore, affects wound healing. MMC can be used intraoperatively or postoperatively. Applying MMC intraoperatively has several advantages over postoperative MMC therapy. The surgeon can control medications, which eliminates the risk of misuse of the toxic chemotherapeutic agent by patients. ${ }^{18}$

However, the mechanism involved in corneo-scleral melt remains unclear. The enzymes produced by polymorphonuclear cells may be involved, leading to the destruction of collagen and proteoglycans that comprise the stroma. ${ }^{19,20}$

Chronic hypoperfusion of the anterior ciliary/episcleral arteries may result in localized/focal anterior segment ischemia and subsequent melting. Neovascularization is an inhibitor of corneal ulceration, ${ }_{1}^{21}$ possibly through the provision of inhibitors of the matrix metalloproteinases (MMPs). ${ }^{22}$ Ischemia and hypoxia result in elevated collagen remodeling in an oxygen-poor environment in the skin; further, unstable collagen molecules are synthesized along with unregulated MMPs, resulting in collagen denaturation, defective angiogenesis, weaker skin, and predisposition to ulceration. ${ }^{23} \mathrm{~A}$ similar mechanism may, therefore, occur in the conjunctiva. In a study conducted using a rabbit model, oxygen therapy was shown to reduce corneal ulceration. ${ }^{24}$ Therefore, ocular ischemia should be considered as a causative or contributing factor to the melting and ulceration of the ocular surface.

When the bare sclera technique is performed in a patient with an otherwise normal ocular surface, epithelialization of the wound area is usually completed within 7-14 days. ${ }^{25,26}$ The epithelium of the operated area should be intact in order to prevent scleral melting when $\mathrm{MMC}$ is used. To prevent scleral melting, the conjunctiva overlying technique is generally, but not always, performed. ${ }^{27,28}$

Considering the findings described above, the patient's ocular conditions were not desirable for a healthy ocular surface. In the eye-whitening surgeries, MMC was 
administered and the bare sclera technique was used without conjunctiva covering; this resulted in ischemia because the regrowth of blood vessels was prohibited. In addition, MMC was administered both intraoperatively and postoperatively.

Generally, surgical procedures are performed for medical and/or cosmetic reasons. The results can vary among individuals primarily due to differences in the healing responses. However, patients' expectations are set high, which drive them to undergo repeated surgeries.

Future studies that collect the long-term postoperative follow-up data of such patients are warranted.

\section{References}

1. Dartt DA. Control of mucin production by ocular surface epithelial cells. Exp Eye Res 2004;78:173-185.

2. Huang LC, Jean D, Proske RJ, et al. Innate immunity at the ocular surface: spectrum of antimicrobial peptide expression. Invest Ophthalmol Vis Sci 2005;46(Suppl):883.

3. Schotveld JH, Beerthuizen JJG, Zaal MJW. Scleral melting in a patient with carotid artery obstruction. Cornea 2006;25:101-103.

4. Sharma A, Gupta A. Bandyopadhyay S, et al. Necrosis of the eyelids and sclera after retrobulbar anaesthesia. J Cataract Refract Surg 2003;29:842-824.

5. Stewart RM, Quah SA, Nguyen DQ, Kaye SB. Conjunctival-corneal melt in association with carotid artery stenosis. Clin Ophthalmol 2008;2:649-655.

6. Alzagoff Z, Tan TD, Chee SP. Necrotising scleritis after bare sclera excision of pterygium. $\mathrm{Br} \mathrm{J}$ Ophthalmol 2000;84:1050-1052.

7. Tsai YY, Lin JM, Shy JD. Acute scleral thinning after pterygium excision with intraoperative mitomycin C: A case report of scleral dellen after bare sclera technique and review of the literature. Cornea 2002;21:227-229.

8. Eckardt C, Bacskulin A. Vitrectomy in intermediate uveitis. Dev Ophtahlmol 1992;23:232-238.

9. Lyne AJ, Lloyd-Jones D. Necrotizing scleritis after ocular surgery. Trans Ophthalmol Soc UK 1979;99:146-149.

10. Reynolds MG, Alfonso E. Infectious scleritis and keratoscleritis: management and outcome. Am J Ophthalmol 1991;112;543.

11. O'Donoghue E, Lightman S, Watson S. Surgically induced necrotising sclerokeratitis: precipitating factors and response to treatment. Br J Ophthalmol 1992;76:17- 21.

12. Maza SM, Foster CS. Necrotizing scleritis after ocular surgery. A clinicopathologic study. Ophthalmol 1991;98:1720-1726.

13. Lin CP, Shih MH, Tsai MC. Clinical experiences of infectious scleral ulceration: A complication of pterygium operation. Br J Ophthalmol 1997;81:980-983.

14. Gungor IU, Ariturk N, Beden U, Darka O. Necrotizing scleritis due to varicella zoster infection: a case report. Ocul Immunol Inflamm 2006;14:317-319.

15. Shome D, Jain V, Jayadev C, et al. Scleral necrosis in a patient with aplastic anaemia. Eye 2007;21:1017.

16. Goodman LS, Gilman A, Goodman Gilman A (Eds.), The pharmacological basis of therapeutics, 8th ed. Elmsford, New York: Pergamon Press 1990; pp. 1247-1248.

17. Verweij J, Pinedo HM. Mitomycin C: mechanism of action, usefulness and limitations. Anticancer Drugs 1990;1:5-13.

18. Oguz H, Basar E, Gurler B. Intraoperative application versus postoperative mitomycin C drops in pterygium surgery. Acta Ophthalmol Scand 1999;77:147-150.

19. Schotveld JH, Beerthuizen JJ, Zaal MJ. Scleral melting in a patient with carotid artery obstruction. Cornea 2006;25:101-103.

20. Seo KY, Lee HK, Kim EK, Lee JH. Expression of tumor necrosis factor alpha and matrix metalloproteinase-9 in surgically induced necrotizing scleritis. Ophthalmic Res 2006;38:66-70. 
21. Conn H, Berman M, Kenyon K, Langer R, Gage J. Stromal vascularization prevents corneal ulceration. Invest Ophthalmol Vis Sci 1980;19:362-370.

22. Fini ME, Cook JR, Mohan R. Proteolytic mechanisms in corneal ulceration and repair. Arch Dermatol Res 1998;290(Suppl):S12-S23.

23. Dalton SJ, Whiting CV, Bailey JR, Mitchell DC, Tarlton JF. Mechanisms of chronic skin ulceration linking lactate, transforming growth factor-beta, vascular endothelial growth factor, collagen remodeling, collagen stability, and defective angiogenesis. J Invest Dermatol 2007;127:958-968.

24. Sharifipour F, Zamani M, Idani E, Hemmati AA. Oxygen therapy for severe corneal alkali burn in rabbits. Cornea 2007;9:1107-1110.

25. Frucht-Pery J, Ilsar M, Hemo I. Single dosage of mitomycin C for prevention of recurrent pterygium: preliminary report. Cornea 1994;13:411-413.

26. Frucht-Pery J, Siganos CS, Ilsar M. Intraoperative application of topical mitomycin C for pterygium surgery. Ophthalmology 1996;103:674-677.

27. Tsai YY, Jane ML, Jium DS. Acute scleral thinning after pterygium excision with intraoperative mitomycin C. Cornea 2002;21:227-229.

28. Abraham S, Igor K, Frederick DR, David L, Joseph FP. Long term effects of mitomycin C in pterygium surgery on scleral thickness and the conjuctiva epithelium. Ophthalmology 2004;111:1522-1527. 